\title{
Performance of an aerogel threshold particle identification detector read out by Hybrid Photon Detectors
}

D. Boutigny, I. De Bonis, J. Favier, Y. Karyotakis, R. Lafaye

L.A.P.P. CNRS/IN2P3, Anecy-le Vieux, France

M. Foucher, H. Jawahery

University of Maryland, College Park, Maryland, USA

E. Borsato, F. Dal Corso, F. Iacovella, M. Morandin, M. Posocco, R. Stroili, E. Torassa and C. Voci

Universita' di Padova and INFN, Sezione di Padova, Italy

$$
\text { J. Oyang }
$$

Califomia Institute of Technology, Pasadena, Califomia, USA

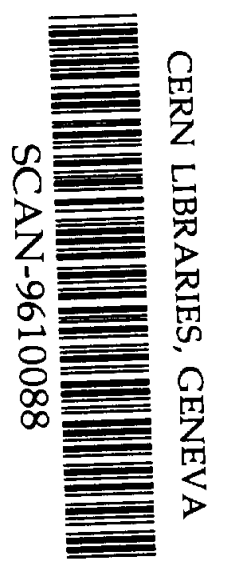

A. Buzykaev, S. Ganzhur, E. Kravchenko,

A. Onuchin, A. Shamov, V. Telnov

Budker Institute of Nuclear Physics, Novosibirsk, Russia

A. Buccheri, F. Ferroni, M. A. Mazzoni, S. Morganti, G. Piredda, R. Santacesaria

Universita' di Roma "La Sapienza" and INFN, Sezione di Roma 1, Italy

(Submitted to the IEEE Transactions on Nuclear Science)

DFPD/96/EP/14

\section{UNIVERSITÀ DEGLI STUDI DI PADOVA}

DIPARTIMENTO DI FISICA "GALILEO GALILEI"

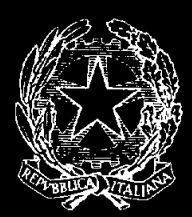

ISTITUTO NAZIONALE DI FISICA NUCLEARE

SEZIONE DI PADOVA 


\title{
Performance of an aerogel threshold particle identification detector read out by Hybrid Photon Detectors
}

\author{
D. Boutigny, I. De Bonis, J. Favier, Y. Karyotakis, R. Lafaye \\ L.A.P.P. CNRS/IN2PS, Annecy-le-Vieux, France \\ M. Foucher, H. Jawahery \\ University of Maryland, College Park, Maryland, USA \\ E. Borsato, F. DalCorso, F. Iacovella, M. Morandin, \\ M. Posocco, R. Stroili, E. Torassa and C. Voci \\ Universila di Padova and INFN, Sezione di Padova, Italy \\ J. Oyang \\ California Institute of Technology, Pasadena, California, USA \\ A. Buzykaev, S. Ganzhur, E. Kravchenko, \\ A. Onuchin, A. Shamov, V. Telnov \\ Budker Institule of Nuclear Physics, Novosibirsk, Russia \\ A. Buccheri, F. Ferroni, M.A. Mazzoni, S. Morganti, \\ G. Piredda, R. Santacesaria \\ Universilá di Roma "La Sapienza" and INFN, Sezione di Roma 1, Ilaly
}

\begin{abstract}
This paper describes an aerogel threshold particle identification detector developed for use in BABAR, the experiment to be run at the asymmetric $e^{+} e^{-} B$-factory PEP-II under construction at the Stanford Linear Accelerator Center (SLAC). Results of extensive tests performed at the CERN PS indicate that this detector is capable of separating pions from below thresiold particles in a wide momentum range. The detector is read out by a new version of a Hybrid Photon Detector (HPD). The HPD has been chosen because of its excellent behavior in high mag. netic fields where it can be successfully exploited to preserve the required PID perfornance.
\end{abstract}

\section{INTRODUCTION}

$\mathrm{T}$ $\mathrm{HE}$ growing interest in $\mathrm{B}$ physics has given new impetus to the development of particle identification (PID) detectors. The separation of kaons from pions plays a key role in tagging the flavor of the neutral $B$ mesons and the identification of interesting final states that are crucial to the measurement of $C P$ violation and CKM parameters.

One of the techniques recently taken into consideration [1], [2], [3] is the detection of Cherenkov light produced by particles crossing aerogel blocks of suitable refraction index.

Due to current availability of low index, good quality aerogel, proof of principle of an aerogel particle identification detector have been established through several beam tests of prototype counters readout with fine mesh photomultipliers in a strong magnetic field up to $2.0 \mathrm{~T}[4],[5],[6],[7]$. With the newly developed proximity-focused Hybrid Photon Detector (HPD), it is now conceivable to design compact detectors that can distinguish pions from kaons up to $\sim 4 \mathrm{GeV} / \mathrm{c}$, simply by measuring the quantity of emitted photons. Such Aerogel Threshold Counters (ATC) are thus much simpler to build and maintain than both traditional ring-imaging detectors and gas-filled Cherenkov counters. They can also find a place inside the high magnetic fields of collider detectors, provided that a suitable photon detector is chosen.

Hereafter we describe the results of tests performed at CERN on an ATC detector read out by proximityfocused HPDs. The HPD version used by us is the latest development of a photon detecter that has recently prompted much work [8] and is here exploited in one of its first applications in a real detector. 


\section{THE EXPERIMENTAL SETUP}

\section{A. Aerogel blocks}

The detector proposed for $B_{A B} B_{A R}[4]$ has to separate pions from kaons between 0.6 and $4.0 \mathrm{GeV} / \mathrm{c}$. This task can be accomplished by an ATC detector formed from two cells of different indices of refraction. The detector arranoement on the heam line is shown in Fioure 1.

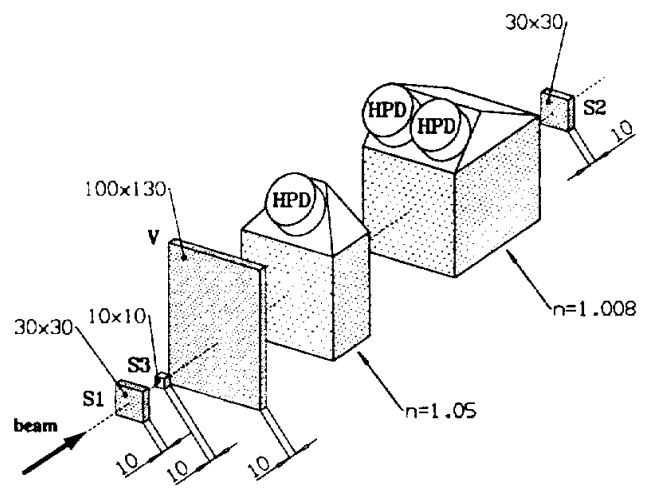

Fig. 1. Schematic view of the detector test beam setup (dimensions are in $\mathrm{mm}$ ).

The first is a block formed from eight $5 \times 5 \times 3$ $\mathrm{cm}^{3}$ slices of aerogel "SAN-95" [9] produced at the Novosibirsk Institute of Catalysis with nominal refraction index $n=1.05$, while the second is a $n=1.008$ block formed from five $9.5 \times 9.5 \times 2.6 \mathrm{~cm}^{3}$ slices produced at the Jet Propulsion Laboratory (JPL). Their overall dimensions and nominal thresholds for $\pi$ and $K$ are reported in Table I.

TABLE I

Properties of AEROGEL USED IN TESTS.

\begin{tabular}{|c|c|c|c|}
\hline $\mathrm{n}$ & $\begin{array}{c}\text { dimensions } \\
\left(\mathrm{cm}^{3}\right)\end{array}$ & $\begin{array}{c}p_{t h r}^{\pi} \\
(\mathrm{GeV} / \mathrm{c})\end{array}$ & $\begin{array}{c}p_{t h r}^{k} \\
(\mathrm{GeV} / \mathrm{c})\end{array}$ \\
\hline 1.05 & $10 \times 10 \times 6$ & 0.44 & 1.54 \\
1.008 & $9.5 \times 9.5 \times 13.1$ & 1.10 & 3.90 \\
\hline
\end{tabular}

These blocks were placed in plastic boxes with 5 faces covered with reflective material while the sixth was an air light guide supporting the photodetector. On the high index block we used a kind of wrapping for all faces and light guide walls consisting of a $50 \mu \mathrm{m}$ layer of aluminum and three layers of $250 \mu \mathrm{m}$ PTFE (Teflon).On the low index block we used the same standard wrapping, except for the three faces of the box parallel to the particle direction. On these faces the inner layer of PTFE was covered with PMP [10], a wavelength shifter that absorbs Cherenkov UV light and re-emits it in the $400 \mathrm{~nm}$ region. The use of PMP is expected to result in improved light collection efficiency, by the fact that it shifts the photon spectrum to longer wavelengths, where the absorption lenght of aerogel is longer.

On the low index block we also used other wrappings, in order to better comprehend the behavior of the detector. All these wrappings had a first layer of $50 \mu \mathrm{m}$ of aluminum and, on top of it:

- WR1: all the 5 faces covered with three layers of $250 \mu \mathrm{m}$ PTFE;

- WR2a: front face with one layer of $250 \mu \mathrm{m}$ PTFE, the remaining 4 faces covered with three layers of 250 $\mu \mathrm{m}$ PTFE;

-WR2b: front and rear faces with one layer of 250 $\mu \mathrm{m}$ PTFE, the remaining 3 faces covered with three layers of $250 \mu \mathrm{m}$ PTFE;

- WR3: same as high index box except front and rear faces covered with 2 layers of Millipore paper ${ }^{1}+2$ layers of Bristol paper ${ }^{2}+1$ layer of PTFE, the remaining 3 faces covered with three layers of $250 \mu \mathrm{m}$ PTFE.

The high index block is viewed by one HPD, while two HPDs are employed for the lower index block that produces fewer photons.

\section{B. Beam setup}

The test was performed at the secondary $\mathrm{T} 10$ beam line of the PS accelerator at CERN during a 3-week run in November and December 1995. Both the sign and momentum of the extracted particles can be selected by the user: negative settings results in a $\pi^{-}$beam while the steering of positive particles selects a beam of $\pi^{+}$and protons with a comparable intensity at 5 $\mathrm{GeV} / \mathrm{c}$. Magnet current settings enable selection of momentum in the range $1-5 \mathrm{GeV} / \mathrm{c}$.

At the end of the extracted beam line, two Gas Cherenkov Counters ( $\mathrm{C} 1$ and $\mathrm{C} 2)$ filled with $\mathrm{CO}_{2}$ with a maximum overpressure of 3.3 bar provided a clean $\pi /$ proton separation for momentum greater than 2.5 $\mathrm{GeV} / \mathrm{c}$.

The experimental set-up was installed at the nominal beam focus position at the end of $\mathrm{C} 1$. Three small plastic scintillator counters (S1,S2,S3 in Figure 1) define the beam trigger.

To avoid triggers with more than one particle crossing the aerogel counters, the beam intensity was optimized and a $10 \times 13 \times 1 \mathrm{~cm}^{3}$ plastic scintillator (V) was used as an out-of-time particle veto counter. The active area of this counter fully covers the active area of the aerogel counters being tested.

To perform a scan over the entire aerogel volume, the prototype detector was placed on top of a motordriven table whose position could be shifted in steps of a few tenths of a millimeter along the horizontal $(x)$ and vertical $(y)$ directions ( $z$ being the beam axis).

\section{The trigger}

Three triggers, causing an event to be read out by the data acquisition system, were implemented: beam, pedestal and LED. The beam trigger was used to select any particle within the beam that passed through the apparatus. We collected data with both positively and

\footnotetext{
${ }^{1}$ Millipore HAWP 0.45 (450 nm), $150 \mu \mathrm{m}$ thick

${ }^{2}$ White paper, $250 \mu$ thick
} 
negatively charged beams. We also collected events in which no particle passed through the apparatus. These were called "pedestal" events. LED events were used to calibrate the photo-detectors.

A beam event was generated by the coincidence of the 3 scintillation counters shown in Figure 1,

$$
\text { beam } \equiv \mathrm{S} 1 \cdot \mathrm{S} 2 \cdot \mathrm{S} 3
$$

A "pedestal" event was triggered by a pulser to monitor the stability of the detectors when there was no input signal. A pulser was also used to activate a light emitting diode (LED), which was connected to the aerogel/photo-detector boxes via an optical fiber. This allowed the photo-detectors to be exposed to a controlled amount of light. The pulse was also sent to the trigger electronics to read out the event. LED events were only collected in separate calibration runs and not as part of normal beam data acquisition procedures.

The final trigger signal sent gating signals to the readout electronics and subsequently caused the event to be recorded.

The T10 beam in the PS provided a $400 \mathrm{~ms}$ burst of particles every $13 \mathrm{~s}$. During each burst we recorded about 50 events of beam particles, and about $5-10$ pedestal events, whereas additional 5 - 10 pedestal events were detected outside the burst.

For some of the data collected we also included the 2 gas Cherenkov counters, in anti-coincidence in the beam trigger. This was to select only protons in the beam.

\section{The DAQ system}

Signals from the HPDs, after being amplified and shaped, were digitized by both flash Analog to Digital Converters (ADC) ${ }^{3}$ and peak-sensing $A D C{ }^{4}$. The sampling frequency of the flash ADC (FADC) was set to $10 \mathrm{MHz}$ for a total sampling period of $15 \mu \mathrm{s}$ while peak sensing was activated by a $1 \mu$ s gate signal. Two flash ADC channels were used to register also the history of the veto counter pulses and the trigger signal timing. Moreover, all the scintillator and beam Cherenkov counter signals were digitized by chargesensing $A D C{ }^{5}$.

In the analysis described here we took the more conservative approach of using only peak-sensing data. A comparison with the performance achievable with the FADC is presented at the end of this paper.

The data acquisition system that was set up to perform the CAMAC and VME readout made use of a CES FIC8232 running OS9, while data recording and real-time analysis procedures were performed on a DEC VMS server connected via ethernet to the FIC. A CES VIC8250 and a VCC2117 allowed transparent access to CAMAC.

${ }^{3} \mathrm{CAEN} V 534,8 \mathrm{ch} ., 8$ bit flash $A D C$

${ }^{4}$ Ortec $916 \mathrm{~A}, 8$ ch., 11 bit peak sensing ADC

${ }^{5}$ LeCroy $2249 \mathrm{~A}, 12 \mathrm{ch}$., 10 bit charge sensing $A D C$
Data acquisition software was based on an available system developed for the KLOE and ALICE test beams [11]. We used the OS9 version for the VME readout. A subset of the recording and monitoring features which usually run on UNIX systems in the present version were ported to VMS, making it possible to perform a thorough quality check during data acquisition, using the CERN standard software packages HBOOK and PAW.

\section{ThE HPD}

The operating principle of the proximity-focused Hybrid Photon Detector (HPD) is shown in Figure 2. Light striking on a $2 \mathrm{~mm}$ thick quartz window hits an S20 UV photo-cathode that features a high quantum efficiency response peaked in the UV region ( $\sim 30 \%$ at $\lambda=300 \mathrm{~nm})$. Photoelectrons are consequently accelerated by a uniform electric field and penetrate in the depleted region of a $300 \mu \mathrm{m}$ silicon diode operated in reverse-bias mode.

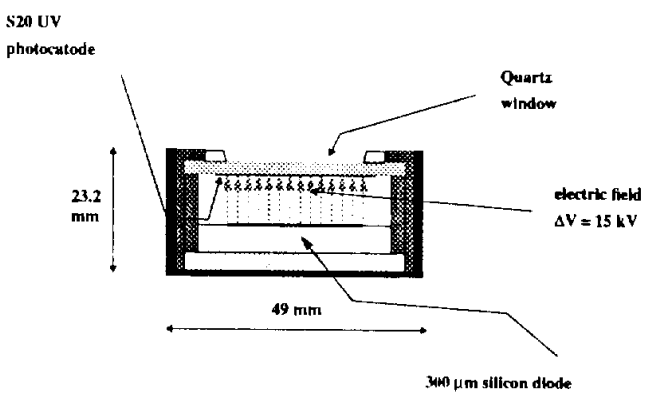

Fig. 2. Schematic diagram of the Hybrid Photon Detector.

The resulting charge pulse can be picked up at the diode contacts. Its amplitude is thousands of times larger than the original photoelectron charge produced on the photo-cathode, since most of the kinetic energy taken on by the photoelectrons is converted into free charges in the silicon at a rate of one electron-hole pair per $3.6 \mathrm{eV}$. The electric output signal is subsequently amplified and conveniently shaped to be measured with the most favorable $\mathrm{S} / \mathrm{N}$ ratio.

Due to uniformity of photoelectron trajectories, the proximity-focused HPD is insensitive to magnetic fields nearly aligned with the axis of the HPD ( [12], [13]). Besides this, the HPD shows negligible degradation of the primary photoelectron statistics which is not the case for other photon detectors like the fine mesh Photo-multiplier.

The HPDs employed in the detector described here ${ }^{6}$ were designed in collaboration with DEP $^{7}$ to meet the requirements of the $B_{A} B_{A R}$ environment and featured the following characteristics:

\footnotetext{
${ }^{6}$ type PP0350, serial no. N9541063, N9541071 and N9541062

${ }^{7}$ DEP, Dwazziewegen 2, 9300 AB Ruden, The Netherlands
} 
- large diode and photo-cathode diameters (1") to improve light collection;

- small dimensions $(23.2 \mathrm{~mm}$ height by $49 \mathrm{~mm}$ diameter) to minimize dead spaces and amount of material; - low diode leakage currents (5-15 $\mathrm{nA}$ range at full depletion) to reduce intrinsic electronic noise.

The HPDs were operated at $15 \mathrm{kV}$, yielding a gain of about 3300 .

\section{A. Amplification and shaping of the HPD signal}

The HPD diode reaches full depletion at about 30 $\mathrm{V}$; in this condition it exhibits a capacitance of about $250 \mathrm{pF}$ and an inverse leakage current in the range of 5-15 nA. The capability to detect the lowest produced signal (single photo-electron) relies on the availability of an amplification chain well suited to these parameters. Moreover, the shaping time $\tau$ of the signal is limited by the background rate expected in a real collider environment. For the ATC detector proposed in $B_{A} B_{A R}$, Monte Carlo simulations show that $\tau$ should not exceed $1 \mu \mathrm{s}$.

Guided by these requirements we designed the preamplifier shown in Figure 3

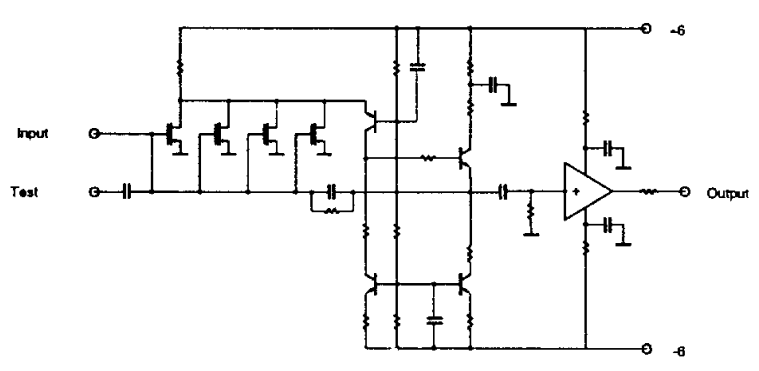

Fig. 3. Schematic diagram of the pre-amplifier circuit.

In order to reduce the series component of the noise, we increased the transconductance of the input device as much as possible by using four 2SK300 FETs in parallel, for a total transconductance of about $80 \mathrm{mS}$. In this way we got a series coefficient noise of $1.8 e^{-} / \mathrm{pF}$ at $1 \mu$ s shaping time.

The circuit is mounted on a small printed circuit board directly attached to the back of the HPD, and is connected to a commercial shaping amplifier with adjustable gain and shaping time via a coaxial cable. The amplifier is a 5 -complex-pole semi-Gaussian shaper ${ }^{8}$ [14], whose peaking time is 2.4 times the nominal shaping time.

Total gain of the pre-amplifier is $1.5 \mathrm{~V} / \mathrm{pC}$ while that of the amplification chain amounts to $120 \mathrm{~V} / \mathrm{pC}$. Typical ATC signals range between 0.5 and $10 \mathrm{fC}$ and therefore produce output signals between $60 \mathrm{mV}$ and $1.2 \mathrm{~V}$, which are well below the saturation limit of the shaper $(100 \mathrm{fC})$.

\footnotetext{
${ }^{8}$ Silena $7612 / \mathrm{L}$
}

\section{B. HPD calibration and performance}

To assess the uniformity and stability of this newly developed HPD, a pre-production of four samples was undertaken and resulted in a successful batch that satisfied our requirements. Thorough evaluation of the performance was accomplished and the results are reported elsewhere [15].

The most important outcome is the rather low noise level $(0.24 \div 0.30$ p.e. corresponding to about $800 \div$ $\left.1000 e^{-}\right)$achieved under test beam conditions. Setting the shaping time at a value of $2 \mu$ s for the best $S / N$ ratio, enabled amplitude spectra like the one reported in Figure 4 to be obtained. The peaks correspond to an increasing number of collected photoelectrons, the leftmost peak representing the pedestal.

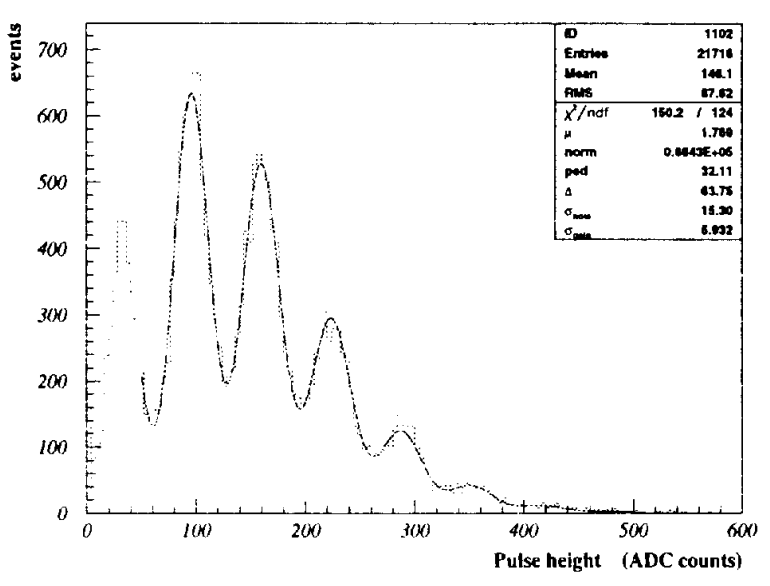

Fig. 4. Pulse height distribution for a LED calibration run.

These distributions, produced by pulsing the device with very short $L E D$ flashes, are well described by the normalized distribution of equation 1 :

(1)

$$
\begin{aligned}
& g(A D C)=\sum_{n=0}^{N} \mu^{n} \frac{e^{-\mu}}{n !}\left[\frac{1}{\sqrt{2 \pi} \sigma_{n}} e^{-\frac{(A D C-(\text { ped }+n \Delta))^{2}}{2 \sigma_{n}^{2}}}\right] \\
& \text { (2) } \quad \sigma_{n}=\sqrt{\sigma_{\text {noise }}^{2}+n \sigma_{\text {gain }}^{2}}
\end{aligned}
$$

where :

$$
\begin{array}{ll}
\mu & =\text { mean number of photoelectrons } \\
A D C & =\text { ADC counts } \\
\text { ped } & =\text { pedestal offset } \\
\Delta & =\text { separation of adjacent peaks } \\
\sigma_{\text {noise }} & =\text { electronic noise } \\
\sigma_{\text {gain }} & =\text { HPD gain spread (1 p.e.) }
\end{array}
$$


From a fit to the spectrum, the parameters can be simultaneously determined and the calibration constant $\Delta$ is then calculated within $1 \%$ accuracy.

In Figure 4 the ratio $\sigma_{\text {noise }} / \Delta$ is equal to 0.24 photoelectrons, representing a level of electronic noise significantly better than that of previous measurements [13]. The achievement of such good noise conditions made it possible to employ a shorter shaping time (1 $\mu \mathrm{s})$ without spoiling the intrinsic performance of the detector.

\section{RESULTS ON THE LOW INDEX AEROGEL}

\section{A. Response to $\beta=1$ particles}

We measured the refraction index of our counter, fitting the momentum dependence of the response using pions in the range between 1 and $1.5 \mathrm{GeV} / \mathrm{c}$, and we found a value compatible within errors with the nominal value $n=1.008$. A $5 \mathrm{GeV} / \mathrm{c}$ pion therefore releases $95 \%$ of what is expected by $\beta=1$ particles. The pulse height recorded in the counter central position is shown in Figure 5. The two HPD signals are summed and the scale is given in numbers of photoelectrons using the calibration computed from the LED run. The mean number of observed photoelectrons is $\mu=8.6 \pm 0.2$.

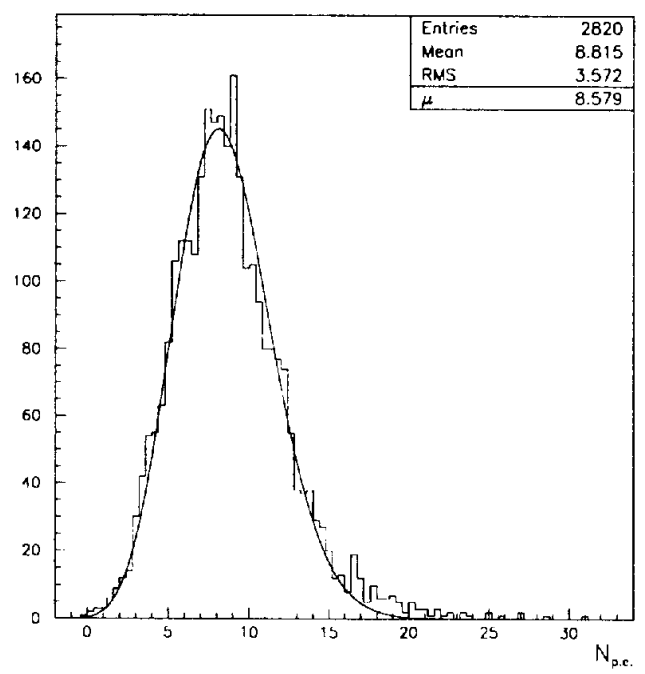

Fig. 5. Number of photoelectrons observed in the low-index aerogel counter read-out by two HPD's for a $5 \mathrm{GcV} / \mathrm{c}$ pion beam at the counter center.

The counter response was measured over its area to study the uniformity of response. Figure 6 presents the results of the scan, normalized to the value observed at the center which was set to 100 . Results show that the response is rather uniform with some enhancement near the side walls due to PMP. For comparison, in Figure 7 we show the results (normalized to the PMP case) of a scan performed under the same conditions, but with no PMP present on the side walls.

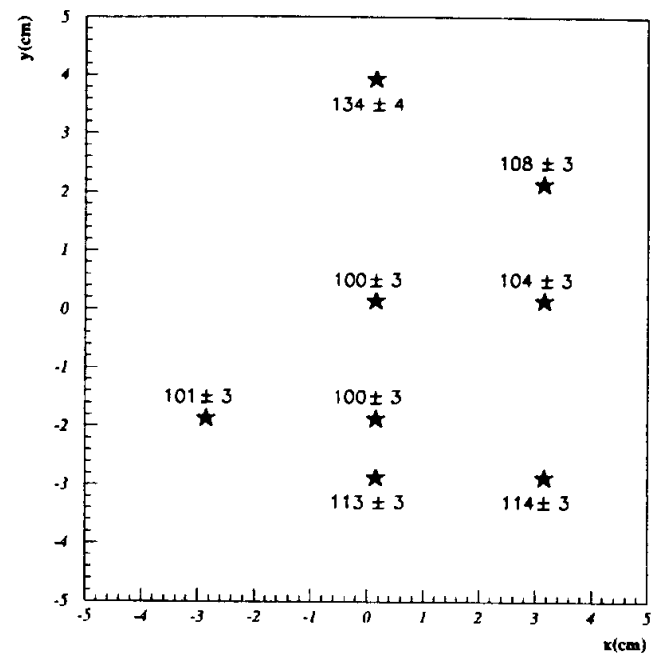

Fig. 6. Results of the counter scan. The values are normalized to the central one. Positive $y$ values are on the light-guide side.

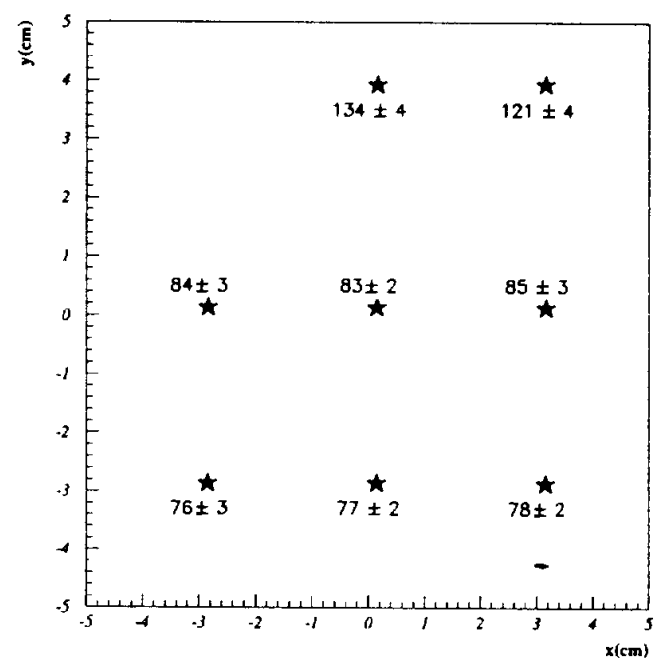

Fig. 7. Results of the counter scan without PMP on the side walls. All values are normalized to the central one obtained with PMP. Positive $y$ values are on the light-guide side.

\section{B. Response to below-threshold particles}

We also studied the response of the counter to below-threshold particles in order to assess the capability of the system to discriminate between pions and kaons of momentum around $4 \mathrm{GeV} / \mathrm{c}$. As in the PS T10 beam there were too few kaons we used $5 \mathrm{GeV} / \mathrm{c}$ protons collected at the same time as the pions and anti-tagged by the two beam Cherenkov gas counters. The surviving kaon component is eliminated with the help of an aerogel counter of refractive index $n=1.012$ $\left(p_{t h r}^{K}=3.2 \mathrm{GeV} / \mathrm{c}\right)$ read out by a standard photomultiplier mounted along the beam line. In principle, 
the signal produced by particles below threshold can be attributed to several different sources:

- Cherenkov light produced in the PTFE wrapping;

- scintillation light produced in aerogel and in PTFE;

- $\delta$ rays;

- electronic noise and discharges;

- beam-related noise;

- contamination by out-of-time particles.

Electronic noise is measured in pedestal runs and the additional beam-related noise is evaluated by comparing pedestals taken in and out of the burst period. The contamination by out-of-time particles is minimized at the analysis stage by vetoing any particle recorded other than the one which started the trigger system. This is performed by the scintillator $V$ (shown in Figure 1), which covers the detector (HPDs included) cross section. All the events accompanied by an extra particle seen by $\mathrm{V}$ in a time window of $(-10 \div 5) \mu \mathrm{s}$ with respect to the trigger are rejected.

In order to evaluate the contribution of light emitted by the particle itself (both in crossing the PTFE layers or scintillating in aerogel) we make a rough assumption that signals below two photoelectrons originate prevailently from this source. We therefore make a fit to this part of the spectrum using equation 1 . We use single tubes rather than the sum to minimize the resolution effects on the single photo-electron peak produced by electronic noise. The results are shown in Figure 8 for both tubes.
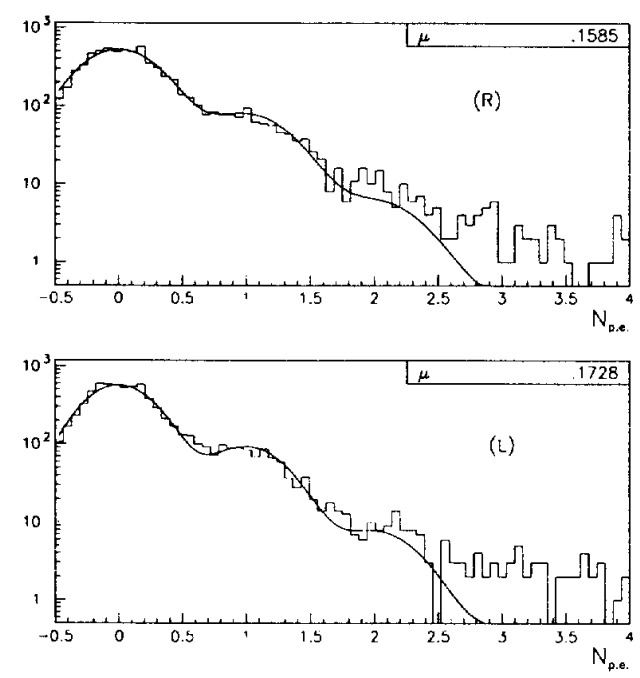

Fig. 8. Pulse-height distribution in the two HPD tubes reading the low index aerogel block. The fit covers the first two photoelectrons.

It is seen that on average the proton light, summed over the two HPDs, is of the order of $1 / 3$ of a photoelectron. Compared to the light released by pions in the same run we conclude that the ratio of light collected from protons alone to pions is $\simeq 0.04$ out of a total background of 0.075 . We attribute the contribu- tion at higher pulse height entirely to $\delta$ rays. We have measured the same quantity using protons at $1 \mathrm{GeV} / \mathrm{c}$ as a cross-check for our assumptions. We expect that neither Cherenkov light from PTFE nor $\delta$ rays should give significant contributions at this momentum. Indeed we measure much less light: the number of events with a recorded pulse height larger than 2 (2.5) photoelectrons is a factor $3.2(7)$ less than in the standard case. These values are upper limits, since kaons at this momentum give some light and cannot be rejected.

\section{Efficiency and Contamination Results}

The results on pions and protons are combined to estimate the performance of the detector in terms of separation power. We compute the following quantities as a function of a varying threshold cut:

- the probability $\rho(p \rightarrow \pi)$ that a $5 \mathrm{GeV} / \mathrm{c}$ proton gives a greater signal than the threshold ;

- the probability $\rho(\pi \rightarrow p)$ that a $5 \mathrm{GeV} / c$ pion gives a signal smaller than the threshold.

These results are summarized in Figure 9. Four pairs of curves are shown, each pair belonging to a run with a different average number of photoelectrons observed for pions. They span the range from the worst condition (poor wrapping, no PMP, most unfavorable beam position) to the reference one (standard wrapping, PMP, center of block). For each pair one can select the threshold which is more convenient for a specific application. At the crossing point $\left(p^{*}\right), p(\pi \rightarrow p)$ becomes equal to $\rho(p \rightarrow \pi)$ and this common probability value may be assumed as a single number expressing the quality of the detector. Moreover the distance from $p^{*}$ to the peaks of the $p$ and $\pi$ signal distributions can be evaluated in units of the relevant standard deviations $(\sigma)$ providing yet another unique number. Hence, we define separation power as twice this value.

From Figure 9 we can see that a threshold can be put between 1.6 and 3.2 photoelectrons, depending on the light yield of the aerogel block. In Table II we summarize the results obtained.

TABLE II

FIGURE OF MERIT OF THE DETECTOR FOR THE $\pi / p$ SEPARATION AT $5 \mathrm{GEV} / \mathrm{C}$.

\begin{tabular}{|c|c|c|}
\hline$N_{\text {p.e. }}^{\pi}$ & $p^{*}(\%)$ & $N_{\sigma}$ \\
\hline \hline 5.2 & 5.9 & 3.1 \\
\hline 6.9 & 4.2 & 3.4 \\
\hline 7.5 & 3.7 & 3.6 \\
\hline 8.6 & 3.3 & 3.7 \\
\hline
\end{tabular}

\section{Effects of wrapping}

We have measured the relative light collection efficiencies for different wrappings, as described in section II-A. Results are presented in Table III.

In the same table the ratio of the light given by particles below threshold $\left(L_{p}\right)$ to that given by $\beta=1$ 


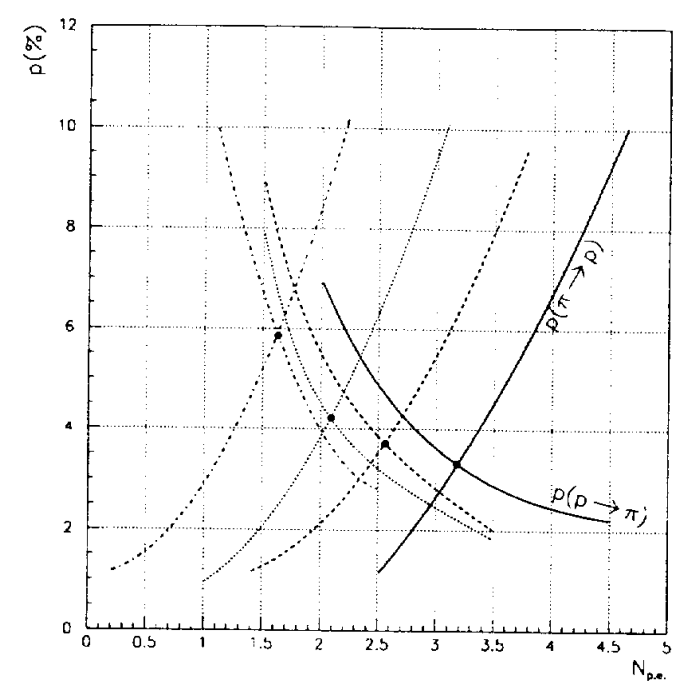

Fig. 9. $p(\pi \rightarrow p)$ and $p(p \rightarrow \pi)$ as a function of the threshold, expressed as the number of photoelectrons for four different cases. Dash-dotted lines represent the observed average number of photoelectrons $(\mu)$ for pions $\mu=5.2$, dotted lines for $\mu=6.9$, dashed lines for $\mu=7.5$ and solid lines for $\mu=8.6$

\section{TABLE III}

LIGHT COLLECTION EFFICIENCY COMPARED TO THE STANDARD CASE (3 PTFE LAYERS + PMP) FOR DIFFERENT WRAPPINGS AS EXPLAINED IN SECTION II-A.

\begin{tabular}{|c|c|c|}
\hline Wrapping & Relative L.Y. (\%) & $L_{p} / L_{\pi}(\%)$ \\
\hline \hline Std. & 100 & $4.2_{-0.5}^{+0.2}$ \\
\hline WR1 & $82 \pm 2$ & $4.6_{-0.5}^{+0.2}$ \\
\hline WR2a & $70 \pm 2$ & $3.7_{-0.4}^{+0.2}$ \\
\hline WR2b & $73 \pm 2$ & $4.5_{-0.5}^{+0.2}$ \\
\hline WR3 & $78 \pm 2$ & $3.0_{-0.3}^{+0.2}$ \\
\hline
\end{tabular}

particles $\left(L_{\pi}\right)$ is also reported. It is possible to note that three layers of PTFE give the highest light yield, although Millipore paper (WR3) can, if necessary, be considered as a valid replacement. Attempts to decrease $L_{p}$ by reducing the front and/or rear PTFE thickness (WR2a, WR2b) do not seem to produce a significant effect and they also diminish the light yield $L_{\pi}$

\section{E. Effects of the PMP}

Besides the desired effect of enhancing the light yield for particles above threshold, the presence of a PMP layer in the wrapping can result in an increase of light production by particles below threshold when they cross the lateral walls of the aerogel block. Although in a pointing geometry detector this is not a serious limitation, we estimated this effect by collecting two special runs with the aerogel block set at an angle of about $45^{\circ}$ with respect to the beam direction and at a transverse position so that particles cross 1 or 2 PMP layers respectively. We expect an additional effect to be due to the light produced mainly by scintillation in PMP. Comparing these runs with the one performed under standard running conditions, we found this contribution to be $0.66 \pm 0.06$ photoelectrons for normal incidence on the PMP surface. This is comparable to the light produced by a $\beta=1$ particle crossing $1 \mathrm{~cm}$ of low index aerogel which is about 0.58 photoelectrons in standard conditions. The net effect on the separation capability of such a detector strongly depends on the particular geometrical arrangement.

\section{RESULTS ON THE HIGH INDEX AEROGEL}

We performed measurements scanning the $10 \times 10$ $\mathrm{cm}^{2}$ area of the high refraction index $(\mathrm{n}=1.05)$ aerogel block using $5 \mathrm{GeV} / \mathrm{c}$ negative pions.

The distribution for a beam run in terms of ADC counts is given in Figure 10.

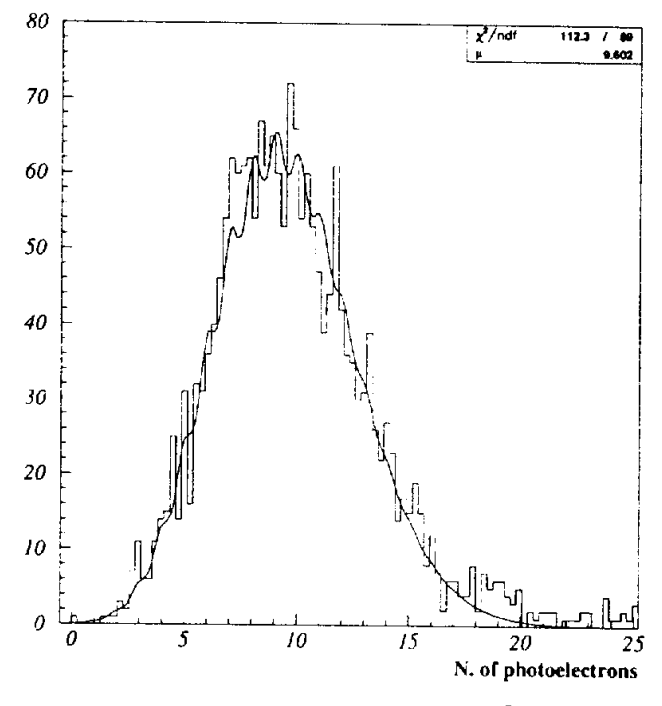

Fig. 10. Pulse height distribution for the high index block with $5 \mathrm{GeV} / \mathrm{c}$ pions.

Figure 11 shows the number of photoelectrons when scanning the aerogel surface with a step of about $4 \mathrm{~cm}$ in $x$ and $y$. As in Figures 6 and 7, positive $y$ values are on the light guide side. We found more than 9 photoelectrons everywhere with a significant signal increase in the region closer to the photodetector while there is no significant dependence on $x$.

As previously done for the high index block, a special run with protons at $p=1 \mathrm{GeV} / \mathrm{c}$ was performed to evaluate the light due to particles below Cherenkov threshold in aerogel.

The number of photoelectrons due to particles under threshold was found to be $0.20 \pm 0.06$ photoelectrons. This information together with the average number of photoelectrons for particles above threshold $(10.76 \pm 0.10)$ makes it possible to extract the contamination and inefficiency percentages. Figure 12 


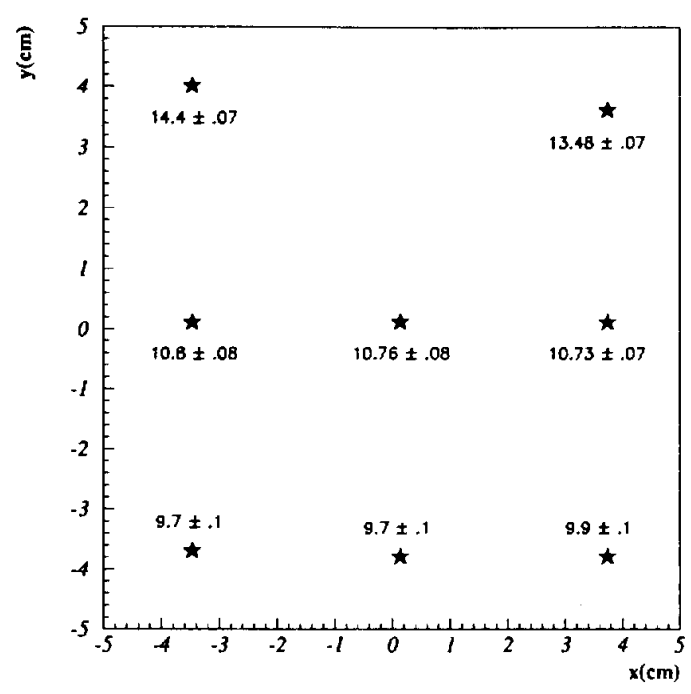

Fig. 11. Number of photoelectrons obtained by scan of the a high-index counter. Statistical and systematic contributions to the uncertainty are separately indicated.

shows contamination and inefficiency depending on the threshold expressed in number of photoelectrons. The crossing point is at $2.0 \%$ with a 2.3 photoelectron threshold that corresponds to a separation of $4.1 \sigma$.

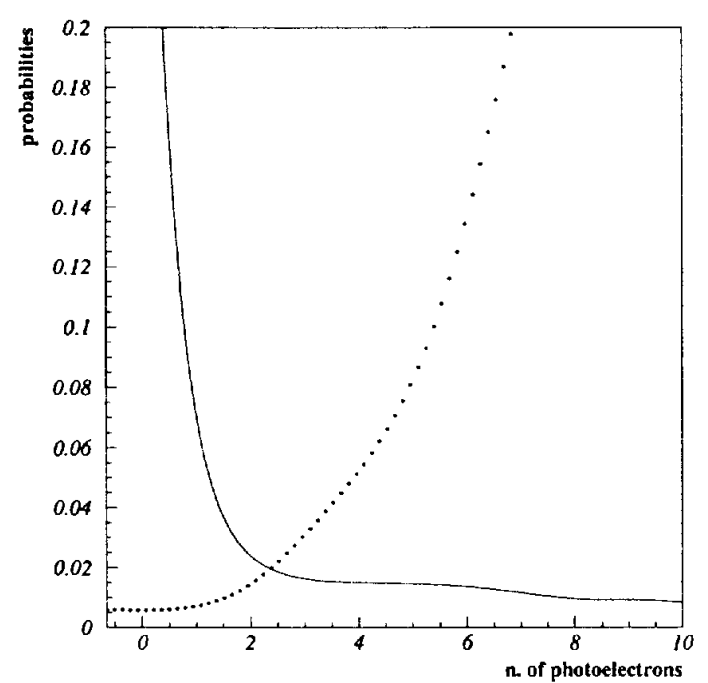

Fig. 12. Contamination and inefficiency curves for the high index aerogel block.

\section{Performance of FADC sampling}

To reduce the signal pile-up due to the high background foreseen in the B-factory environment, the shaping time chosen for the read-out electronics is 1 $\mu \mathrm{s}$ which is not the optimal one $(2 \mu \mathrm{s})$ for achieving the best resolution in the amplitude measurement. However, by sampling the signal waveform with a flash

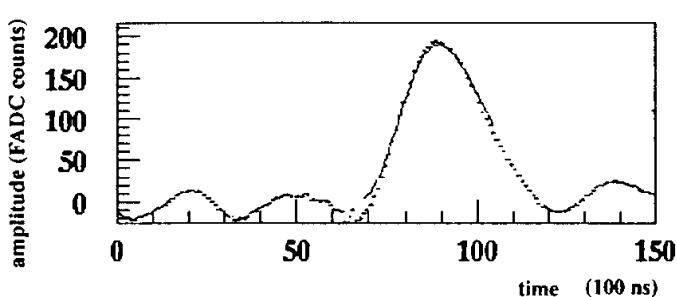

Fig. 13. FADC event: ADC value versus time ticks (in hundreds of ns).

$A D C$, it is possible to recover the accuracy of the peak sensing ADC obtained with optimal shaping time [16].

The FADC data (of which one event is shown in Figure 13) were analyzed by fitting an appropriate function on the samples. The function used to fit the sampled signal is the following [17]:

(3)

$$
\begin{aligned}
f(t)= & A \times\left(\frac{K_{1}}{e^{\tau_{0}\left(t-t_{0}\right)}}+\right. \\
& \frac{K_{2} \cos \left(\omega_{1}\left(t-t_{0}\right)\right)+K_{3} \sin \left(\omega_{1}\left(t-t_{0}\right)\right)}{e^{\tau_{1}\left(t-t_{0}\right)}}- \\
& \left.\frac{K_{4} \cos \left(\omega_{2}\left(t-t_{0}\right)\right)+K_{5} \sin \left(\omega_{2}\left(t-t_{0}\right)\right)}{e^{\tau_{2}\left(t-t_{0}\right)}}\right)
\end{aligned}
$$

where the coefficients $K_{i}, \tau_{0}, \tau_{1}, \tau_{2}, \omega_{1}, \omega_{2}$ are determined by the shaping circuit characteristics [14] and by the chosen shaping time, while $A$, the amplitude of the signal, and $t_{0}$, the time when the particle crossed the detector, are suitably fitted.

The resolution $\left(\sigma_{1} / \Delta\right.$, where $\sigma_{1}$ and $\Delta$ are defined in equation 2) on the single photoelectron (see Figure 14) is improved from 0.30 p.e (obtained with the peak- sensing ADC) to 0.24 p.e., which is the best resolution which could be obtained with a peak-sensing ADC using the optimal shaping time value of $2 \mu s$. Note that in this figure the first peak corresponds to the first photoelectron, as the pedestal is removed in the fitting procedure, while in Figure 4 the first peak corresponds to the pedestal peak. In Figure 14 the curve obtained by fitting equation 1 on the distribution is also shown.

An important reason for using a FADC instead of a peak-sensing $A D C$ is that rejection of background due to out-of-time particles is improved. Figure 15 shows the behavior of the time resolution as a function of the amplitude of the signal (expressed as the number of photoelectrons). As the threshold cut-off point for rejecting below-threshold particles is $>2$ photoelectrons, the expected time resolution is better than $200 \mathrm{~ns}$.

The data are fitted with a curve that is inversely proportional to the amplitude. 


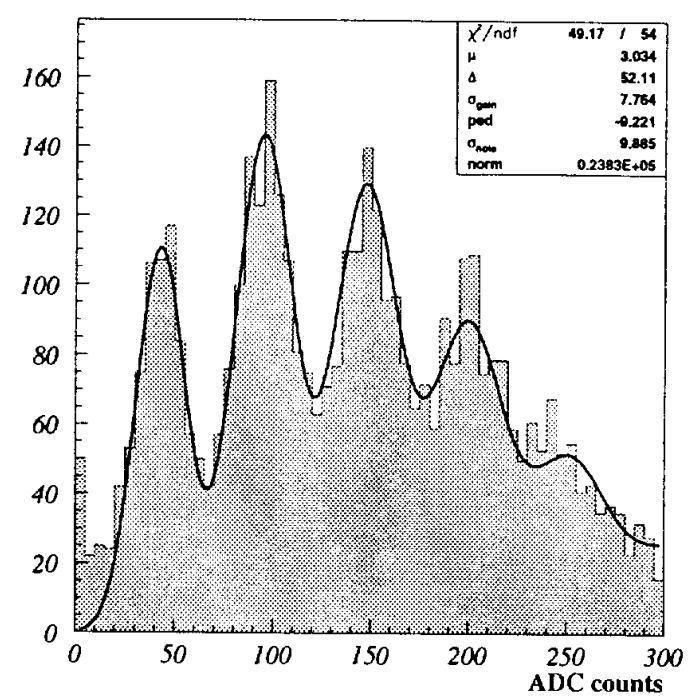

Fig. 14. Pulse height distribution obtained from the FADC data with a superimposed fit of equation 1 .

\section{Comparison With M.C. Simulations}

A Montecarlo based on GEANT-3.21 [18] was developed to simulate the data. A special code was used for treatment of Rayleigh scattering of optical photons, which is not currently simulated in GEANT but is essential in a realistic simulation of light propagation in aerogel. The simulation program needs as input a detailed description of the aerogel absorption and scattering lengths, of the PTFE wrapping reflectivity and the HPD quantum efficiency as functions of the photon wavelength. As almost no measurements of the above first three quantities existed for the two aerogel blocks when the test was performed, some crude assumptions were used. A $96 \%$ reflectivity, independent from the photon energy, was assumed for the PTFE wrapping, while the HPD quantum efficiency was tabulated according to manufacturer's specifications.

For the low-index simulation, the absorption length was taken to be proportional to $\lambda^{6}$ that looks compatible with previous measurements of aerogel blocks of various refraction indices, while the scattering length was taken to be proportional to $\lambda^{4}$, as expected. The absolute normalizations were tuned with the data of the scan without PMP.

The same parametrizations were also used in the PMP case, where the effect of PMP was simulated by using the absorption efficiency and the emission spectrum measured in a laboratory test (see Figure 16).

The high-index aerogel simulation requires reduction of the absorption and scattering lengths by a factor of almost two and a further ad hoc strong suppression of photons with a wave length below $300 \mathrm{~nm}$.

Results of the simulations are compared with data in Figures 17 and 18.

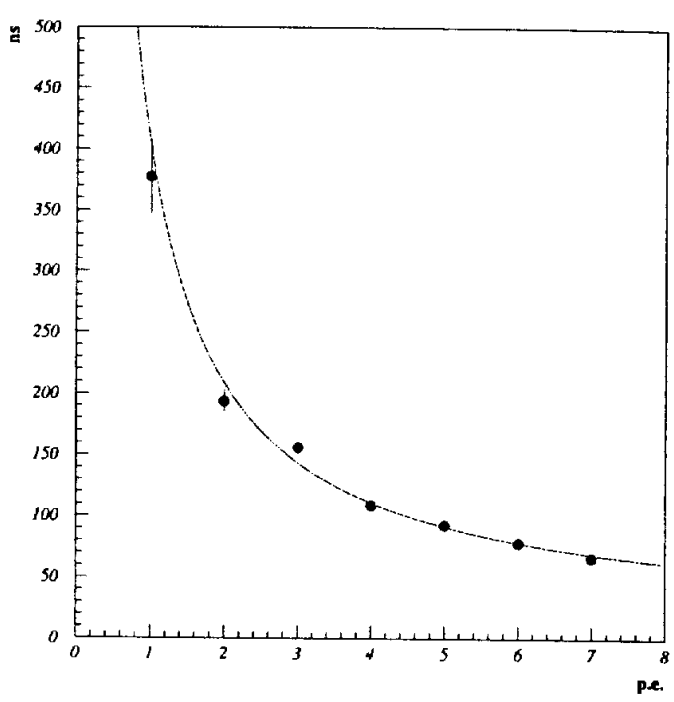

Fig. 15. Time resolution (in ns) as a function of the signal amplitude; in abscissa the amplitude is expressed in terms of the number of photoelectrons.
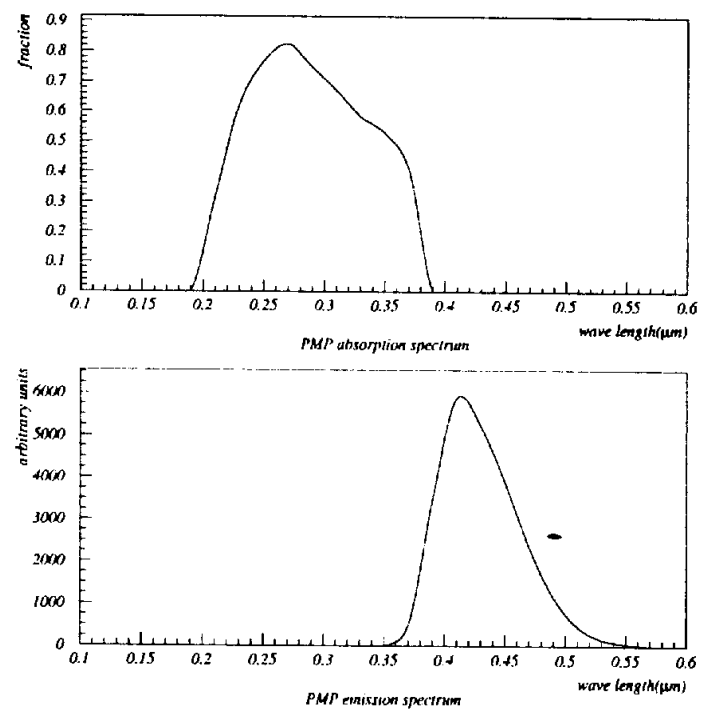

Fig. 16. PMP absorption efficiency and emission spectrum

As new measurements of the PTFE wrapping [19] and of the high index aerogel absorption and scattering lengths [9] were recently performed by some of us and other measurements are in progress, a complete simulation of the test data with more realistic parameters is foreseen in the next future. 


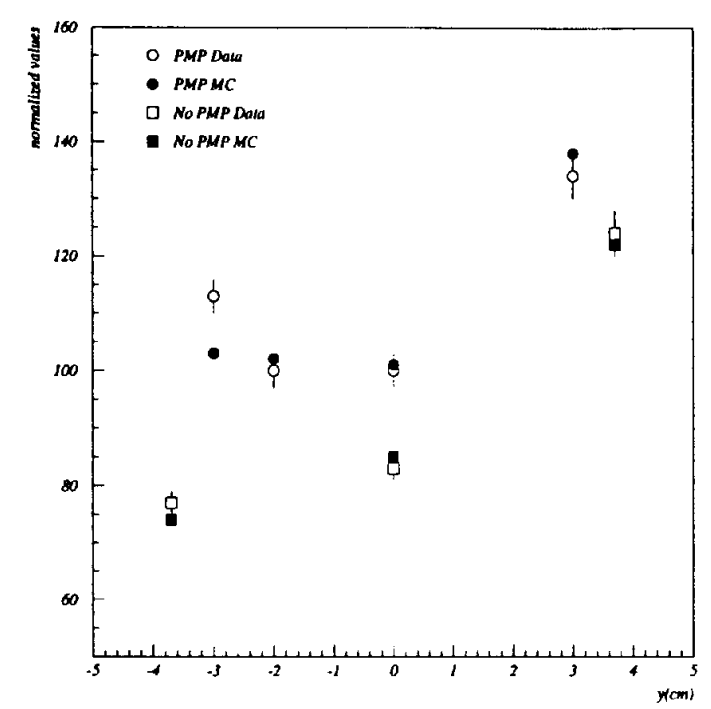

Fig. 17. Low-index aerogel MC comparisons to data with and without PMP at $x=0$.

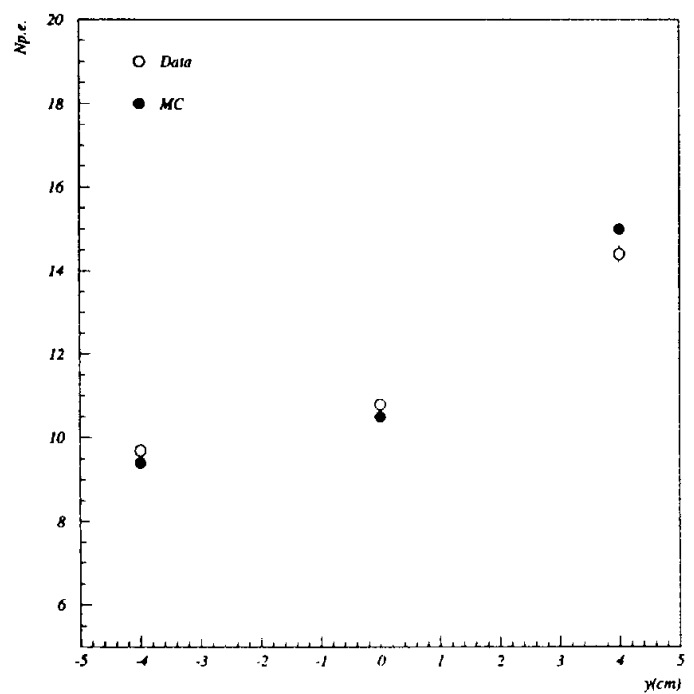

Fig. 18. High-index aerogel MC comparison to data at $\mathrm{x}=-\mathbf{3 . 5}$. 


\section{Conclusions}

We have studied the performance of a threshold aerogel counter system for separating kaons from pions in the momentum range from 0.6 to $4.0 \mathrm{GeV} / \mathrm{c}$ in a test beam. This was the prototype of the $B_{A} B_{A R}$ PID system in the forward region. Since this detector is meant to operate in a $1.5 \mathrm{~T}$ field, the read-out was performed by HPD tubes. This, to our knowledge, has been one of the first implementations of such tubes in a real detector. The main results achieved are:

- HPD tubes proved to be a reliable detector well suited to detect the feeble light pulses produced in aerogel counters;

- PTFE appears to be the best material for wrapping the aerogel in order to obtain the highest light collection efficiency;

- PMP wavelength shifter helps to further increase the light collection efficiency, its effect has been studied in some detail;

- the performance of the system is reasonably understood and can be reproduced by a MC simulation tuned to the data, allowing predictions as to the effects of small changes in the system.

We have shown that a PID system based on a silica aerogel threshold counter is a simple, sturdy, yet efficient device. Our conclusion, based on the experimental results of this test and on their mild MC extrapolation, is that a system very similar to the one described in this paper can accomplish the task of separating kaons from pions in the momentum range from 0.6 to $4.0 \mathrm{GeV} / \mathrm{c}$.

\section{ACKNowledgments}

We are greatly grateful to P. Tsou and L. Hrubesh for their R\&D effort of producing high quality aerogel blocks and their continuous support since 1992. The fruitful collaboration of the Padova INFN mechanical team led by C. Fanin has been of invaluable help for the preparation of the ATC prototype. We are especially grateful to G. Maccato for his continuous and thoughtful support. We are also indebted with G. Mazzi for his helpful assistance in performing the measurements of the PMP optical properties. It is finally a pleasure to acknowledge the hospitality of the CERN PS Division.

\section{REFERENCES}

[1] D.G. Hitlin G. Eigen and J. Oyang, "Silica aerogel Cherenkov detectors for particle identification," oct 1992 , CALT-68-1829.

[2] "B factories; the state of the art in accelerators, detectors, and physics," Nov. 1992, SLAC-400.

[3] "Status report on the design of a detector for the study of CP violation at PEP-II at SLAC," Jun. 1993, SLAC-419.

[4] D. Boutigny et al., "Technical Design Report," Tech. Rep., SLAC, Mar. 1995, SLAC-R-95-457.

[5] J. Oyang et al., BaBar note 137 \& 142.

[6] A. Onuchin et al., Nucl. Inst. and Melh., vol. A 315 , pp. 517-520, 1992.

[7] A. Buzykaev et al., BaBar note 170.
[8] G. Anzivino et al., Nucl. Inst. and Meth., vol. A 365, p. 76, 1995.

[9] A. Buzykaev et al., "Aerogel for the Cherenkov counters," Nucl. Inst. and Meth., vol. A 379, 1996.

[10] P. Schuster H. Güsten and W. Seitz, J. Phys. Chem., vol. 82, p. $459,1978$.

[11] H. Beker, http://hpbeker.roma1.infn.it/rio.

[12] S. Basa et al., Nucl. Inst. and Meth., vol. A 330, p. 93 , 1993.

[13] M. Calvi et al., Nucl. Inst. and Meth., vol. A 369, p. 157, 1996.

[14] S. Ohkawa et al., Nucl. Inst. and Meth., vol. 138, p. 85, 1976.

[15] E. Borsato et al., "Extensive tests of Hybryd Photon Detectors (HPD) used to collect Cherenkov light," presented at the First Conference on new developments in photodetection, Beaune, 1996.

[16] W. E. Cleland et al., Nucl. Inst. and Meth., vol. 338, p. 467, 1994.

[17] Silena Spa, Spetroscopy Amplifier mod. 7612 User Guide.

18] R. Brun at al., GEANT 9.21, CERN, 1994.

[19] A. Buzykaev et al., "Project of Aerogel Cherenkov Counters for KEDR," Nucl. Inst. and Meth., vol. A 379, 1996. 
$\therefore$

- 